\title{
New mechanism producing axions in the AQN model and how the CAST can discover them
}

\author{
H. Fischer* \\ University of Freiburg, Freiburg 79104, Germany \\ X. Liang ${ }^{\dagger}$ and A. Zhitnitsky ${ }^{\ddagger}$ \\ Department of Physics \& Astronomy, University of British Columbia, Vancouver V6T1Z1, Canada \\ Y. Semertzidis ${ }^{\S}$ \\ Center for Axion and Precision Physics Research, Institute for Basic Science, \\ Daejeon 34141, Republic of Korea \\ and Department of Physics, KAIST, Daejeon 34141, Republic of Korea \\ K. Zioutas \\ Physics Department, University of Patras, Patras GR 26504, Greece
}

(Received 8 June 2018; published 15 August 2018)

\begin{abstract}
We advocate the idea that there is a fundamentally new mechanism for the axion production in the Sun, which has never been discussed previously in the literature. This novel mechanism of the axion production is based on the so-called sxion quark nugget $(\mathrm{AQN})$ dark matter model. These axions will be produced in addition to wellstudied axions emitted due to the Primakoff effect. The AQN model was originally invented as a natural explanation of the observed ratio $\Omega_{\text {dark }} \sim \Omega_{\text {visible }}$ when the DM and visible matter densities assume the same order of magnitude values, irrespective to the axion mass $m_{a}$ or initial misalignment angle $\theta_{0}$. This model, without adjustment of any parameters, reproduces reasonably the intensity of the extreme UV (EUV) radiation from the solar corona as a result of the AQN annihilation events with the solar material. This extra energy released in the corona represents a resolution, within the AQN framework, a long-standing puzzle known in the literature as the "solar corona heating mystery." The same annihilation events also produce the relativistic axions. This represents a new mechanism of the axion production and constitutes the main subject of this work. The flux of these axions is unambiguously fixed in this model and expressed in terms of the EUV luminosity from the corona. We also compute the spectral properties of these axions and make a few comments on the potential for the discovery of these solar axions by the upgraded CAST (CERN Axion Solar Axion) experiment.
\end{abstract}

DOI: $10.1103 /$ PhysRevD.98.043013

\section{INTRODUCTION}

The Peccei-Quinn (PQ) mechanism accompanied by the axions remains the most compelling explanation of the strong $C P$ problem; see original papers [1-3] and recent reviews [4-12] on the subject. We refer to the review articles for discussions and analysis on the recent activities in the field of the axion searches by a number of different groups using very different instruments.

\footnotetext{
*Horst.Fischer@cern.ch

†unyul@phas.ubc.ca

\#arz@physics.ubc.ca

\&annis@ibs.re.kr

"Konstantin.Zioutas@cern.ch
}

Published by the American Physical Society under the terms of the Creative Commons Attribution 4.0 International license. Further distribution of this work must maintain attribution to the author(s) and the published article's title, journal citation, and DOI. Funded by SCOAP .
For the purposes of the present work, it is sufficient to mention that the conventional dark matter galactic axions are produced due to the misalignment mechanism [13] when the cosmological field $\theta(t)$ oscillates and emits cold axions before it settles down at its final destination $\theta_{\text {final }}=0$. Another mechanism is due to the decay of the topological objects [14-18]. There are a number of uncertainties and remaining discrepancies in the corresponding estimates. We shall not comment on these subtleties ${ }^{1}$ by referring to the original papers [14-18]. It is important that in both cases the

\footnotetext{
${ }^{1}$ According to the most recent computations presented in Ref. [18], the axion contribution to $\Omega_{\mathrm{DM}}$ as a result of decay of the topological objects can saturate the observed DM density today if the axion mass is in the range $m_{a}=(2.62 \pm 0.34) 10^{-5} \mathrm{eV}$, while the earlier estimates suggest that the saturation occurs at a larger axion mass. One should also emphasize that the computations [14-18] have been performed with assumption that PQ symmetry was broken after inflation.
} 
produced axions are nonrelativistic particles with typical $v_{\text {axion }} / c \sim 10^{-3}$, and their contribution to the dark matter density scales as $\Omega_{\text {axion }} \sim m_{a}^{-7 / 6}$. This scaling unambiguously implies that the axion mass must be fine-tuned $m_{a} \simeq$ $10^{-5} \mathrm{eV}$ to saturate the DM density today, see footnote 1 , while larger axion mass will contribute very little to $\Omega_{\mathrm{DM}}$. The cavity type experiments have a potential to discover these nonrelativistic axions.

Axions can be also produced as a result of the Primakoff effect in a stellar plasma at high temperature [19]. These axions are ultrarelativistic as the typical average energy of the axions emitted by the Sun is $\langle E\rangle=4.2 \mathrm{keV}$, see [20]. Today the most sensitive broadband searches for solar axions come from the helioscope CAST at CERN [20].

The main goal of the present work is to argue that there is a fundamentally novel mechanism of the axion production in the Sun. This mechanism is rooted to the so-called axion quark nugget (AQN) dark matter model. We overview the basic ideas of this model in Sec. II. Meanwhile, we want to make two important comments related to this model in the context of the axion physics.

First basic consequence of this model is as follows. We already mentioned about two mechanisms on energy transfer from the original cosmological axion field $\theta(t)$ to the DM nonrelativistic axions as a result of the misalignment mechanism and decay of the topological defects. The key element of the present work is that in addition to these two well-established processes there is one more path how the original cosmological field $\theta(t)$ can transfer its energy to the axions. This mechanism is based on the idea that the AQNs might be formed during the same QCD epoch when two other processes of the axion production were operational. This process of formation inevitably includes the closed $N=$ 1 axion domain walls as an essential part of the construction. The corresponding axion energy (hidden in the form of the axion domain wall) is not available unless the AQN itself gets annihilated and destroyed, in which case the axion energy will be released into the space in the form of the free propagating axions which can be observed on Earth.

The second important comment is that these emitted axions will be released with relativistic (but not ultrarelativistic) velocities with typical values $v_{\text {axion }}^{\mathrm{AQN}} \simeq 0.5 c$. These features should be contrasted with conventional galactic nonrelativistic axions $v_{\text {axion }} \sim 10^{-3} c$ and solar ultrarelativistic axions with typical energies $\langle E\rangle=4.2 \mathrm{keV}$.

We highlight the basic logic and the ideas of the AQN dark matter in Sec. II. Now we want to present a few observational hints in context of the solar physics which apparently support this generic AQN proposal.

The AQN model was invented long ago [21] (though a specific formation mechanism of the nuggets was developed in much more recent papers [22-24]) as a natural explanation of the observed ratio $\Omega_{\text {dark }} \sim \Omega_{\text {visible. The }}$ similarity between dark matter $\Omega_{\text {dark }}$ and the visible matter $\Omega_{\text {visible }}$ densities strongly suggests that both types of matter have been formed during the same cosmological epoch, which must be the QCD transition as the baryon mass $m_{p}$ which represents the visible portion of the matter $\Omega_{\text {visible }}$ is obviously proportional to $\Lambda_{\mathrm{QCD}}$, while the contribution related to the $\mathrm{E} \& \mathrm{~W}$ physics proportional to the quark mass $\sim m_{q}$ represents only a minor contribution to the proton mass.

In the context of the present work, the argument supporting the AQN model goes as follows. It has been known for quite some time that the total intensity of the observed EUV (extreme ultraviolet) and (soft) x-ray radiation (averaged over time) can be estimated as follows,

$$
L_{\odot(\text { from Corona) })} \sim 10^{30} \cdot \frac{\mathrm{GeV}}{\mathrm{s}} \sim 10^{27} \cdot \frac{\mathrm{erg}}{\mathrm{s}},
$$

which represents (since 1939) the renowned "solar corona heating puzzle"; see, e.g., a general review [25] on the subject and also Ref. [26] with analysis of some specific features related to present work. The observation (1) implies that the corona has the temperature $T \simeq 10^{6} \mathrm{~K}$ which is 100 times hotter than the surface temperature of the Sun, and conventional astrophysical sources fail to explain the EUV and soft $\mathrm{x}$ ray radiation from corona $[25,26]$.

It turns out that if one estimates the extra energy being produced within the AQN dark matter scenario one obtains the total extra energy $\sim 10^{27} \mathrm{erg} / \mathrm{s}$ which surprisingly reproduces (1) for the observed EUV and soft x-ray intensities [27]. One should add that the estimate $\sim 10^{27} \mathrm{erg} / \mathrm{s}$ for extra energy is derived exclusively in terms of known dark matter density $\rho_{\text {DM }} \sim 0.3 \mathrm{GeV} \mathrm{cm}^{-3}$ and dark matter velocity $v_{\text {DM }} \sim 10^{-3} c$ surrounding the sun without adjusting any parameters of the model; see Sec. III with relevant estimates. The recent numerical Monte Carlo simulations carried out in [28] strongly support this estimation. We interpret this "numerical coincidence" as an additional indication supporting the AQN model. Our original remark relevant for the present work is that if one accepts the explanation $[27,28]$ that the solar corona heating puzzle is resolved within $\mathrm{AQN}$ scenario then the axion flux will be unambiguously fixed in terms of the EUV observed luminosity (1) as the axion field represents the crucial element in the AQN construction.

Another inspiring observation supporting the AQN scenario in the context of the present studies can be explained as follows. It was recently claimed in Ref. [29] that a number of highly unusual phenomena observed in the solar atmosphere can be explained by the gravitational lensing of "invisible" streaming matter towards the Sun, see also [26]. The phenomena include, but not limited to such irradiation as the EUV emission, frequency of $\mathrm{X}$ and $\mathrm{M}$ flare occurrences, etc. Naively, one should not expect any correlations between the flare occurrences, the intensity of the EUV radiation, and the position of the planets. Nevertheless, the analysis [29] obviously demonstrates that this naive expectation is not quite correct. At the same time, the emergence of such 
correlations within AQN framework is a quite natural effect. This is because the dark matter AQNs can play the role of the "invisible" matter in Ref. [29], which triggers otherwise unexpected solar activity sparking also the large flares [30]. Therefore, the observation of the correlation between the EUV intensity and frequency of the flares can be considered as an additional supporting argument of the AQN related dark matter explanation of the observed EUV irradiation (1), because both effects are originated from the same dark matter AQNs. As a direct consequence of this relation we expect that the intensity of the axion emission from the Sun (which always accompanies the EUV emission) will be also correlated with the position of the planets.

The paper is organized as follows. In Sec. II, we overview the AQN model by paying special attention to the astrophysical and cosmological consequences of this specific dark matter model. In Sec. III, we highlight the basic arguments of Ref. [27] advocating the idea that the annihilation events of the antinuggets with the solar material can be interpreted as the nanoflares conjectured by Parker long ago. Precisely these annihilation events emit the axions and we compute the intensity and spectral properties of these axions in Sec. IV. Finally, in Sec. V, we highlight the basic ideas of the design of a new detector and comment on possible potential of the discovery of these axions emitted from the solar corona. We conclude in Sec. VI with a few thoughts on the future development of the solar axion searches.

\section{AXION QUARK NUGGET (AQN) DARK MATTER MODEL}

The axion field plays a key role in the construction. Therefore, we would like to make a short overview of this model with emphasis on the role of the axion field and related astrophysical consequences of this proposal.

The idea that the dark matter may take the form of composite objects of standard model quarks in a novel phase goes back to quark nuggets [31], strangelets [32], nuclearities [33], see also review [34] with large number of references on the original results. The AQN model in the title of this section stands for the axion quark nugget model [21] to emphasize on essential role of the axion field in the construction and to avoid confusion with earlier models [31-34] mentioned above. The AQN model is drastically different from previous similar proposals in two key aspects:

(1) There is an additional stabilization factor in the AQN model provided by the $N=1$ axion domain walls which are copiously produced during the QCD transition.

(2) The AQN could be made of matter as well as antimatter in this framework as a result of separation of charges, see recent papers [22-24] with large number of technical details.
To recapitulate these two important ingredients: the axions play a dual role in construction of the AQNs as they provide an additional pressure to stabilize the nuggets and also play the role of the source (through the axion field) which breaks $\mathcal{P}$ and $\mathcal{C P}$ symmetries during the QCD transition. Precisely these $\mathcal{P}$ and $\mathcal{C P}$ violating processes are responsible for the separation of charges, leading to the fundamental and very generic consequence of this framework expressed as $\Omega_{\text {dark }} \sim \Omega_{\text {visible }}$. The key role of the axion field at present epoch manifests itself as a substantial contribution $(\sim 1 / 3)$ to the total nugget's mass [24].

The basic idea of the AQN proposal can be explained as follows: It is commonly assumed that the Universe began in a symmetric state with zero global baryonic charge and later (through some baryon number violating process, the so-called baryogenesis) evolved into a state with a net positive baryon number. As an alternative to this scenario we advocate a model in which "baryogenesis" is actually a charge separation process when the global baryon number of the Universe remains zero. In this model the unobserved antibaryons come to comprise the dark matter in the form of dense nuggets of quarks and antiquarks in colour superconducting (CS) phase. The formation of the nuggets made of matter and antimatter occurs through the dynamics of shrinking axion domain walls, see original papers [22-24] with many technical details.

The nuggets, after they formed, can be viewed as the strongly interacting and macroscopically large objects with a typical nuclear density and with a typical size $R \sim\left(10^{-5}-10^{-4}\right) \mathrm{cm}$ determined by the axion mass $m_{a}$ as these two parameters are linked, $R \sim m_{a}^{-1}$. This relation between the size of nugget $R$ and the axion mass $m_{a}$ is a result of the equilibration between the axion domain wall pressure and the Fermi pressure of the dense quark matter in CS phase. One can easily estimate a typical baryon charge $B$ of such macroscopically large objects as the typical density of matter in CS phase is only a few times the nuclear density. However, it is important to emphasize that there are strong constraints on the allowed window for the axion mass, which can be represented as follows $10^{-6} \mathrm{eV} \leq m_{a} \leq 10^{-2} \mathrm{eV}$. This axion window corresponds to the range of the nugget's baryon charge $B$ which largely overlaps with all presently available and independent constraints on such kind of dark matter masses and baryon charges

$$
10^{23} \leq|B| \leq 10^{28},
$$

see e.g., $[35,36]$ for review. The corresponding mass $\mathcal{M}$ of the nuggets can be estimated as $\mathcal{M} \sim m_{p} B$, where $m_{p}$ is the proton mass.

This model is perfectly consistent with all known astrophysical, cosmological, satellite and ground-based constraints within the parametrical range for the mass $\mathcal{M}$ and the baryon charge $B$ mentioned above (2). It is 
also consistent with known constraints from the axion search experiments. Furthermore, there is a number of frequency bands where hints for excess of emission exist, but could not be explained by conventional astrophysical sources. Our comment here is that this model may explain some portion, or even entire excess of the observed radiation in these frequency bands, see short review [35] and additional references at the end of this section.

Another key element of this model is the coherent axion field $\theta$ which is assumed to be nonzero during the QCD transition in early Universe. As a result of these $\mathcal{C P}$ violating processes the number of nuggets and antinuggets being formed would be different. This difference is always of order one effect [22-24] irrespectively to the parameters of the theory, the axion mass $m_{a}$ or the initial misalignment angle $\theta_{0}$. As a result of this disparity between nuggets and antinuggets a similar disparity would also emerge between visible quarks and antiquarks. This is precisely the reason why the resulting visible and dark matter densities must be the same order of magnitude [22-24]

$$
\Omega_{\mathrm{dark}} \approx \Omega_{\mathrm{visible}}
$$

as they are both proportional to the same fundamental $\Lambda_{\mathrm{QCD}}$ scale, and they both are originated at the same QCD epoch. If these processes are not fundamentally related the two components $\Omega_{\text {dark }}$ and $\Omega_{\text {visible }}$ could easily exist at vastly different scales.

Another fundamental ratio (along with $\Omega_{\mathrm{dark}} \approx \Omega_{\mathrm{visible}}$ discussed above) is the baryon to photon ratio at present time

$$
\eta \equiv \frac{n_{B}-n_{\bar{B}}}{n_{\gamma}} \simeq \frac{n_{B}}{n_{\gamma}} \sim 10^{-10} .
$$

If the nuggets were not present after the phase transition the conventional baryons and antibaryons would continue to annihilate each other until the temperature reaches $T \simeq 22 \mathrm{MeV}$ when density would be 9 orders of magnitude smaller than observed (4). This annihilation catastrophe, normally thought to be resolved as a result of "baryogenesis" as formulated by Sakharov [37].

In our proposal (in contrast with conventional frameworks on baryogenesis) the annihilation stops because the extra antibaryon charge is hidden in the antinuggets, while the total baryon charge of the Universe remains zero at all times. The ratio (4) in the AQN framework is determined by a single parameter with a typical QCD scale, the formation temperature $T_{\text {form }} \approx 40 \mathrm{MeV}$, slightly lower than the critical temperature $T_{c}$ of the gap in CS phase. This temperature is defined by a moment in evolution of the Universe when the nuggets and antinuggets basically have completed their formation and not much annihilation would occur at lower temperatures $T \leq T_{\text {form }}$, see [22,23] for the details.
Unlike conventional dark matter candidates, such as WIMPs (weakly interacting massive particles) the darkmatter/antimatter nuggets are strongly interacting and macroscopically large objects, as we already mentioned. However, they do not contradict any of the many known observational constraints on dark matter or antimatter in the Universe due to the following main reasons [38]: They carry very large baryon charge $|B| \gtrsim 10^{23}$, and so their number density is very small $\sim B^{-1}$. As a result of this unique feature, their interaction with visible matter is rare, and therefore, the nuggets perfectly qualify as DM candidates. Furthermore, the quark nuggets have very large binding energy due to the large gap $\Delta \sim 100 \mathrm{MeV}$ in CS phases. Therefore, the baryon charge is so strongly bounded in the core of the nugget that it is not available to participate in big bang nucleosynthesis (BBN) at $T \approx 1 \mathrm{MeV}$, long after the nuggets had been formed.

It should be noted that the galactic spectrum contains several excesses of diffuse emission the origin of which is unknown, the best known example being the strong galactic $511 \mathrm{keV}$ line. If the nuggets have the average baryon number in the $\langle B\rangle \sim 10^{25}$ range they could offer a potential explanation for several of these diffuse components. It is important to emphasize that a comparison between emissions with drastically different frequencies in such computations is possible because the rate of annihilation events (between visible matter and antimatter DM nuggets) is proportional to one and the same product of the local visible and DM distributions at the annihilation site. The observed fluxes for different emissions thus depend through one and the same line-of-sight integral

$$
\Phi \sim R^{2} \int d \Omega d l\left[n_{\text {visible }}(l) \cdot n_{\mathrm{DM}}(l)\right],
$$

where $R \sim B^{1 / 3}$ is a typical size of the nugget which determines the effective cross section of interaction between $\mathrm{DM}$ and visible matter. As $n_{\mathrm{DM}} \sim B^{-1}$ the effective interaction is strongly suppressed $\sim B^{-1 / 3}$. The parameter $\langle B\rangle \sim 10^{25}$ was fixed in this proposal by assuming that this mechanism saturates the observed $511 \mathrm{keV}$ line [39,40], which resulted from annihilation of the electrons from visible matter and positrons from antinuggets. Other emissions from different frequency bands are expressed in terms of the same integral (5), and therefore, the relative intensities are unambiguously and completely determined by internal structure of the nuggets which is described by conventional nuclear physics and basic QED, see short overview [35] with references on specific computations of diffuse galactic radiation in different frequency bands.

Finally we want to mention that the recent EDGES (Experiment to Detect the Global Epoch of reionization Signatures) observation of a stronger than anticipated $21 \mathrm{~cm}$ absorption [41] can find an explanation within the AQN framework as recently advocated in [42]. The 
basic idea is that the extra thermal emission from AQN dark matter at early times produces the required intensity (without adjusting any parameters) to explain the recent EDGES observation.

\section{AQNS AS THE CORONA'S HEATERS}

Our goal here is to overview the basic parameters related to the AQNs entering the solar atmosphere from outer space. The impact parameter for capture and crash of the nuggets by the Sun can be estimated as

$$
b_{\text {cap }} \simeq R_{\odot} \sqrt{1+\gamma_{\odot}}, \quad \gamma_{\odot} \equiv \frac{2 G M_{\odot}}{R_{\odot} v^{2}},
$$

where $v \simeq 10^{-3} c$ is a typical velocity of the nuggets. Assuming that $\rho_{\mathrm{DM}} \sim 0.3 \mathrm{GeV} \mathrm{cm}^{-3}$ and using the capture impact parameter (6), one can estimate the total energy flux due to the complete annihilation of the nuggets,

$$
\begin{aligned}
L_{\odot(\mathrm{AQN})} & \sim 4 \pi b_{\text {cap }}^{2} \cdot v \cdot \rho_{\mathrm{DM}} \\
& \simeq 3 \times 10^{30} \cdot \frac{\mathrm{GeV}}{\mathrm{s}} \\
& \simeq 4.8 \times 10^{27} \cdot \frac{\mathrm{erg}}{\mathrm{s}},
\end{aligned}
$$

where we substitute constant $v \simeq 10^{-3} c$ to simplify numerical analysis. This is obviously an order of magnitude estimate as we ignore a large number of factors of order one. ${ }^{2}$ Nevertheless, this order of magnitude estimate is very suggestive as it roughly coincides with the observed total EUV energy output from corona (1) representing $\sim\left(10^{-7}-10^{-6}\right)$ portion of the total solar luminosity. Precisely this "accidental numerical coincidence" was the main motivation to put forward the idea [27] that (7) represents a new source of energy feeding the EUV and soft $\mathrm{x}$-ray radiation. The numerical simulations [28] strongly support the estimate (7) and entire picture of the framework. In particular, the numerical studies [28] show that the annihilation events mostly occur at the altitude close to $2000 \mathrm{~km}$ where the temperature of the plasma $T \simeq 10^{6} \mathrm{~K}$. Therefore, it is quite natural to expect that the most photons emitted from the annihilation events in the environment will have the energies in EUV and soft x-rays bands.

The basic claim of $[27,28]$ is that the annihilation events of the antinuggets, which generate huge amount of energy (7) can be identified with the "nanoflares" conjectured by Parker long ago [43]. In most studies the term "nanoflare"

\footnotetext{
${ }^{2}$ For example, we ignore that only antinuggets, not nuggets generate the energy (7). Furthermore, a large amount of annihilation energy will be emitted in form of the neutrinos. In addition, even $E \& M$ energy released as a result of annihilation might be radiated in different energy spectrum, not necessary in form of EUV radiation. Finally, approximately $1 / 3$ of the energy will be emitted in form of the axions as we shall argue in Sec. IV.
}

describes a generic burst-like event for any impulsive energy release on a small scale, without specifying its cause. In other words, in most studies the hydrodynamic consequences of impulsive heating (due to the nanoflares) have been used without discussing their nature, see review papers [25,44]. The novel element of Ref. [27] is that the nature of the nanoflares was specified as annihilation events of the dark matter particles within AQN framework, i.e.,

$$
\text { nanoflares } \equiv \mathrm{AQN} \text { annihilation events, }
$$

in which case the observed intensity of the EUV (7) is determined by the DM density $\rho_{\mathrm{DM}}$ in the solar system. The main arguments of $[27,28,30]$ supporting the identification (8) and the basic picture in general are:

(1) In order to reproduce the measured radiation loss, the observed range of nanoflares needs to be extrapolated from sub-resolution events with energy $3.7 \times 10^{20}$ erg to the observed events interpolating between $\left(3.1 \times 10^{24}-1.3 \times 10^{26}\right)$ erg. This energy window corresponds to the (anti)baryon charge of the nugget $10^{23} \leq|B| \leq 4 \times 10^{28}$ which largely overlaps with allowed window (2) for AQNs reviewed in Sec. II. This is a highly nontrivial consistency check for the proposal (8) as the window (2) comes from a number of different and independent constraints extracted from astrophysical, cosmological, satellite and ground-based observations. The window (2) is also consistent with known constraints from the axion search experiments within the AQN framework. Therefore, the overlap between these two fundamentally different entities represents a highly nontrivial consistency check of the proposal (8).

(2) The corresponding $E \& M$ radiation is expected to be mostly in form of the EUV and soft x-ray emissions because the annihilation events of the AQNs mostly occur at the altitude around $2000 \mathrm{~km}$ with a typical width around a few hundred kilometers and the typical temperature $T \simeq 10^{6} \mathrm{~K}$. This extra energy injection (7) represents in our framework the explanation of the unusual features of the so-called transition region (TR) when the temperature of the plasma experiences some drastic changes by two orders of magnitude on the length scales of a few hundred kilometers.

(3) Our next argument goes as follows. The nanoflares are distributed very uniformly in quiet regions, in contrast with micro-flares and flares which are much more energetic and occur exclusively in active areas. It is consistent with our identification (8) as the antinugget annihilation events should be present in all areas irrespectively to the activity of the Sun. At the same time the flares, triggered by the AQNs as 
suggested in [30], are originated in the active zones, and therefore cannot be uniformly distributed.

(4) The observed Doppler shifts (corresponding to velocities $250-310 \mathrm{~km} / \mathrm{s}$ ) and the observed line width in OV of $\pm 140 \mathrm{~km} / \mathrm{s}$ far exceed the thermal ion velocity which is around $11 \mathrm{~km} / \mathrm{s}$ as discussed in [27]. These observed features can be easily understood within the AQN scenario. Indeed, the typical velocities of the nuggets entering the solar corona is about $\sim 618 \mathrm{~km} / \mathrm{s}$, the escape velocity of the sun. Therefore, it is perfectly consistent with observations of the very large Doppler shifts and related broadenings of the line widths. Typical time scales of the nanoflare events, of order of $\left(10^{1}-10^{2}\right)$ sec are also consistent with estimates [27].

(5) It has been observed [45] that "the pre-flare enhancement propagates from the higher levels of the corona into the lower corona and chromosphere." It is perfectly consistent with our proposal as the dark matter AQNs enter the solar atmosphere from outer space. Therefore, they first enter the higher levels of the corona where they generate the shock wave, before they reach chromosphere in $\tau \sim\left(10-10^{2}\right)$ sec.

(6) It has been claimed in $[46,47]$ that the observations show the "ubiquitous presence of chromospheric anemone jets outside of sunspots...". In our framework the jet-like structure is a direct consequence of the AQNs entering the solar atmosphere when the nuggets generate the shock waves with large Mach number $M \sim 10$ which represents a typical jetlike structure [30]. One should emphasize here that the most of these events are sub-resolution events which are well below the instrumental threshold $\sim 3 \times 10^{24} \mathrm{erg}$, see item 1 above.

\section{INTENSITY AND THE SPECTRUM OF THE SOLAR AXIONS}

In Sec. II, we explained that the axion field is the key element in the AQN construction. In Sec. III, we argued that the AQNs may serve as the heaters of the corona. In this section, we estimate the intensity and spectral properties of the axions which will be inevitably produced as a result of the annihilation and complete disintegration of the antinuggets in the solar corona.

\section{A. Intensity}

The axions play a key role in construction of the AQNs as they provide an additional pressure to stabilize the nuggets; see Sec. II for review. The corresponding axion contribution into the total nugget's energy density has been computed in [24], see the red curve in Fig. 9 in [24]. Depending on parameters the axion's contribution to the nugget's mass represents about $1 / 3$ of the total mass. It implies that this entire energy will be radiated in form of the free propagating axions. This energy can be expressed in terms of the axion luminosity from the Sun as follows

$$
L_{\odot(\text { axion })} \sim \frac{1}{3} L_{\odot(\mathrm{AQN})} \simeq 1.6 \times 10^{27} \cdot \frac{\mathrm{erg}}{\mathrm{s}}
$$

where $L_{\odot(\mathrm{AQN})}$ is given by (7). The corresponding axion flux measured on Earth can be computed as follows,

$$
\begin{aligned}
\Phi_{\text {axions }} & \sim \frac{L_{\odot(\text { axion })}}{4 \pi\left\langle E_{a}\right\rangle D_{\odot}^{2}} \sim 3 \times 10^{16} \frac{1}{\mathrm{~cm}^{2} \mathrm{~s}}\left(\frac{10^{-5} \mathrm{eV}}{m_{a}}\right), \\
D_{\odot} & \simeq 1.5 \times 10^{13} \mathrm{~cm},
\end{aligned}
$$

where we assume that the axion's energy when the antinuggets get annihilated is slightly relativistic $E_{a} \simeq 1.2 m_{a}$, but never becomes very relativistic, see precise estimates below.

The axion flux (10) should be compared with the flux computed in [20] as a result of the Primakoff effect:

$$
\begin{aligned}
\Phi_{a}(\text { Primakoff }) & \simeq 3.75 \times 10^{11} \frac{\mathrm{g}_{10}^{2}}{\mathrm{~cm}^{2} \mathrm{~s}} \\
\mathrm{~g}_{10} & \equiv \mathrm{g}_{\mathrm{a} \gamma} / 10^{-10} \mathrm{GeV}^{-1}, \\
\langle\mathrm{E}\rangle & =4.2 \mathrm{keV} .
\end{aligned}
$$

The axion flux (10) is much larger than the conventional flux (11). However, the energies of the axions in these two mechanisms are drastically different. Therefore, the energy flux of the conventional flux (11) is also much larger than the axion energy flux due to the nuggets,

$$
\begin{aligned}
m_{a} \Phi_{\text {axions }} & \sim 3 \times 10^{11} \frac{\mathrm{eV}}{\mathrm{cm}^{2} \mathrm{~s}}, \\
\langle E\rangle \Phi_{a}(\text { Primakoff }) & \sim \mathrm{g}_{10}^{2} \times 10^{15} \frac{\mathrm{eV}}{\mathrm{cm}^{2} \mathrm{~s}} .
\end{aligned}
$$

It is very instructive to compare these fluxes with conventional cold dark matter galactic axion contribution assuming the axions saturate the observed DM density:

$$
\begin{aligned}
m_{a} \Phi(\text { galactic axions }) & \sim \rho_{\mathrm{DM}} \cdot v_{\mathrm{DM}} \\
& \simeq \frac{0.3 \mathrm{GeV}}{\mathrm{cm}^{3}} v_{\mathrm{DM}} \\
& \simeq 10^{16} \frac{\mathrm{eV}}{\mathrm{cm}^{2} \mathrm{~s}} .
\end{aligned}
$$

We emphasize that the estimate (9) in this framework is almost model-independent expression as it is directly linked to the observed EUV luminosity (1) and (7). This intimate relation between EUV luminosity and the axion luminosity emerges as a result that both radiations (EUV and the axions) are related to the same physics and occur as a result of the annihilation of the antinuggets in the corona 
in the AQN framework. In contrast, the estimate (10) is a model-dependent result as it is based on our computations of the spectral properties of the axion emission determined by the average $E_{a} \simeq 1.2 m_{a}$. The corresponding estimate is the subject of the next subsection.

\section{B. Spectral properties}

As mentioned earlier in this section, study in [24] indicates that the total energy of an AQN finds its minimum when the axion (domain wall) contributes $1 / 3$ of its total mass. Now consider an AQN loosing its mass when entering the solar corona, such that the axion quickly increases its portion of total mass in comparison with the equilibrium value. One should comment here that the axion domain wall in the equilibrium does not emit any axions as a result of pure kinematical constraint: the static domain wall axions are off-shell nonpropagating axions. The timedependent perturbation obviously changes this equilibrium configuration. In other words, the configuration becomes unstable with respect to emission of the axions because the total energy is no longer at its minimum. To retrieve its ground state, an AQN will therefore intend to lower its domain wall portion of the energy by radiation of axions. To summarize: the emission of axions is an inevitable consequence during the annihilation of antinuggets in the solar corona simply for the reason to maintain the AQN stability.

Now, we want to identify a precise mechanism which produces the on-shell freely propagating axions emitted by the axion domain wall. In this section, we overview the basic idea of the computational technique to be used. We refer to the Appendix for the technical details of the computations. To address this question, we consider the general form of a domain wall solution,

$$
\phi\left(R_{0}\right)=\phi_{w}\left(R_{0}\right)+\chi,
$$

where $R_{0}$ is the radius of the AQN, $\phi_{w}$ is the classical solution of the domain wall, while $\chi$ describes the excitations due to the time-dependent perturbation. We should note that, $\phi_{w}$ is clearly off-shell classical solution, while $\chi$ describes the on-shell propagating axions. Thus, whenever the domain wall is excited, namely $\chi \neq 0$, freely propagating axions may be produced and emitted by the excitation modes.

Suppose an AQN is traveling in vacuum where no annihilation event takes place, we expect the solution stays in its ground state $\phi\left(R_{0}\right)=\phi_{w}\left(R_{0}\right)$ which corresponds to the minimum energy state. Since there is no excitation (i.e., $\chi=0$ ), no free axion can be produced. However, the scenario drastically changes when some baryon charge from the AQN get annihilated. Due to these annihilation processes, the AQN starts to loose a small amount of its mass, and consequentially its size shrinks from $R_{0}$ to a slightly smaller radius $R_{\text {new }}=R_{0}-\Delta R$. Note that its quantum state $\phi\left(R_{0}\right)=\phi_{w}\left(R_{0}\right)$ is no longer the ground state, because a lower energy state $\phi_{w}\left(R_{\text {new }}\right)$ with lower value of $B$ becomes available. Then, we may write the current state of the domain wall as $\phi\left(R_{0}\right)=\phi_{w}\left(R_{\text {new }}\right)+$ $\phi^{\prime}\left(R_{\text {new }}\right) \Delta R$, so the domain wall now has a nonzero exciting mode $\chi=\phi^{\prime}\left(R_{\text {new }}\right) \Delta R$ and free axions can be produced during oscillations of the domain wall.

To reiterate: the annihilation of antinuggets in the solar corona forces the surrounding domain wall to oscillate. These oscillations of domain wall generate excitation modes and ultimately lead to radiation of the propagating axions.

We conclude this section by highlighting the results of the computations. We present our results in this section for the velocity distribution. Equivalently, one can represent the same information in terms of the energy and momentum distribution. We refer to the Appendix for the corresponding technical details.

Normally, it is convenient to express the normalized spectrum as a function of the speed of emitted axion $v_{a} / c$, defines as follows

$$
\begin{aligned}
\rho\left(v_{a}\right) & \equiv \frac{1}{\Phi_{\text {axions }}^{\text {tot }}} \frac{d}{d v_{a}} \Phi_{\text {axions }}\left(v_{a}\right), \\
\Phi_{\text {axions }}^{\text {tot }} & \sim 3 \times 10^{16} \frac{1}{\mathrm{~cm}^{2} \mathrm{~s}}\left(\frac{10^{-5} \mathrm{eV}}{m_{a}}\right), \\
\int_{0}^{1} d v_{a} \rho\left(v_{a}\right) & =1 .
\end{aligned}
$$

In Fig. 1, the we represent the results in the entire region of the allowed kinematical domain, from $v_{a} / c=0$ to 1 . We can see the distribution is roughly Gaussian, and it peaks near $v_{a} / c \sim 0.5$ with a sharp cut at $v / c \gtrsim 0.9$. Thus we would expect the axion flux is relativistic but not ultrarelativistic, in contrast with conventional Primakoff effect (11).

We should comment here that at very small velocities $v_{a} \ll c$ the spectrum shows the linear dependence on $v_{a}$.

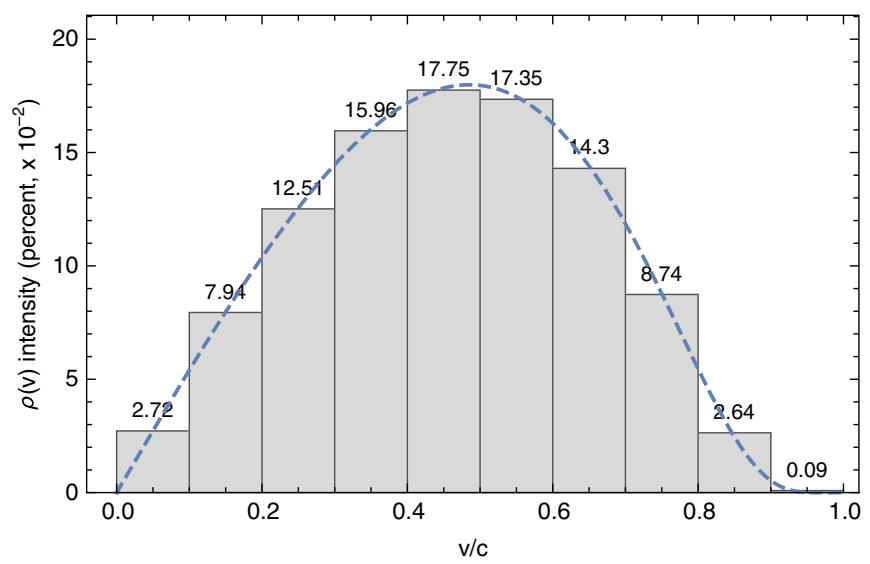

FIG. 1. The normalized spectrum $\rho(v)$ vs $v / c$. The numbers inside the spectrum give the estimated percentage of the intensity per velocity BIN of $0.1 \mathrm{c}$. 
We think that it is an artifact of our computational approximations. Indeed, our technical derivation is based on the "thin-wall approximation"; see the Appendix. That is, assuming the conventional thickness of the domain wall $\sim m_{a}^{-1}$, the thin-wall approximation is justified when $\lambda_{a} \lesssim m_{a}^{-1}\left(\lambda_{a}\right.$ stands for de Broglie wavelength of the emitted axion). While this condition is marginally satisfied in case of an relativistic axion, it is badly violated in nonrelativistic regime. A different computational technique is obviously required to address the question about the spectral properties in nonrelativistic regime. The region of small $v_{a} \leq 0.1 c$ contributes very little (around $\sim 1 \%$ ) to the normalization integral (15). Therefore, it will be ignored for the purposes of the present work.

\section{DESIGN: CAST AND OTHER DARK MATTER AXION ANTENNAE}

In this section, we discuss the discovery potential of the relativistic axions emitted by the AQNs as a result of the annihilation events. We consider separately the solar axions in subsection VA and the axions radiated in the Earth's atmosphere and in the Earth's core in subsection V B.

\section{A. AQNs in the Sun}

The reasoning about this new production of the DM axions of solar origin emerging during the interaction of AQNs with the outer Sun along with the derived velocity spectrum, define their detection scheme. We take here as an example the CAST-CAPP DM axion antenna [48] whose commissioning is scheduled soon. In fact, this antenna is of the Sikivie type, whose design has been supplemented with a fast scanning mode, becoming thus a quasi wide-band DM axion antenna. This modification makes CAST sensitive to streaming DM axions including axion mini-clusters (aMCs), without compromising the conventional search for galactic axions $[49,50]$.

The DM axions from AQN defragmentation in the corona are the same axions with the same coupling constant $f_{a}$ which is the subject of many other axion searches; see reviews [4-12]. However, there are a few differences we want to point out here because these distinct features are important for their detection and identification:

(1) the axions which are produced due to the AQN annihilations in the Sun will be emitted from narrow transition region with width of a few hundred kilometers at the altitude around $2000 \mathrm{~km}$ where the most of annihilation events occur; see Fig. 9 in Ref. [28]. The probability for these axions to be reabsorbed inside the Sun is negligible, similar to the conventional arguments [20] for the axions produced by the Primakoff effect. The sensitivity of available instruments is not likely to resolve this structure at present time. Therefore, as a first rough approximation for the Earth's observable, one can ignore this structure and assume that entire Sun with angular diameter of $0.55^{\circ}$ emits these axions.

(2) these axions are characterized by the relativistic velocities $(v \approx 0.5 \mathrm{c})$; see Fig. 1 . The corresponding distribution is very distinct from the galactic axions with typical velocities of order $v \approx 10^{-3} \mathrm{c}$.

(3) if the AQNs are at the origin of spatiotemporally confined solar activity [27], this will provide a trigger in real time due to the continuous monitoring of the Sun by various observatories. This is unique in the field of the dark sector, and, with Fig. 1 in mind, the warning time will be at least 15 minutes. In addition, this implies an improvement of the signalto-noise ratio, allowing also to distinguish the solar DM component from the galactic one $\left(v \approx 10^{-3} \mathrm{c}\right)$.

In what follows, we would like to consider a specific example of the planning experiments [48] which in principle are capable to detect these axions produced by AQN mechanism. In our estimates below we use the technical characteristics as presented in [48]. The basal solar DM axion flux at the Earth is about 1 per mille compared to that of the galactic axions. The size of the CAST-CAPP cavities require that the de Broglie wavelength of the axions be $\lambda_{\mathrm{dB}} \sim 4$ meters, ensuring phase matching between several cavities. This condition applies to many axion DM experiments (e.g., [48]). The various CAPP cavities can cover an axion rest mass range of about 3 to $120 \mu \mathrm{eV}(0.7$ to $30 \mathrm{GHz})$. The CAST search will start at about $20 \mu \mathrm{eV}$, being thus sensitive to velocities below $0.01 \mathrm{c}$.

One should mention that the DM stream can drastically change the number of annihilating events in the solar atmosphere as argued in [29]. The corresponding changes will lead to drastic temporary variation of the axion flux from the Sun. These drastic changes can be anticipated as the time delay is at least 15 minutes. It gives us a hope to observe such time-dependent short enhancements in the axion flux.

At the same time, a planetary gravitationally focused DM stream of AQNs can change the number of annihilating events in the solar or earth atmosphere by a factor up to $10^{6}$, see Refs. [49,51,52]. Interestingly, in recent work [53], an amplification factor by as much as $1.2 \times 10^{11}$ is given for the Sun as gravitational lens for incident light downstream at about 520 AU. Given the fact that the Einstein ring and the deflection angle increase with velocity $v$ as $v^{-1}$ and $v^{-2}$, respectively, all this improves the situation for an Earth observer searching for nonrelativistic particles with $v \ll c$. Thus, the impact on streaming AQNs during alignment of Earth-Sun with the assumed AQN stream is much larger than the aforementioned planetary impact towards the Sun. This is actually reasonable; i.e., the Sun is of course a better gravitational lens than any planet. Therefore, an axion haloscope like CAST may well profit from such a drastic flux enhancement by the Sun. Moreover, signatures from 
the active Earth atmosphere could be used as axion trigger, which in Ref. [29] have shown even a planetary dependence. More intriguing might be the decades long puzzle of ionization excess in December. We recall, that, on the 18th of December, there will be an alignment Earth-SunGalactic center within $5.5^{\circ}$.

The recent theoretical and numerical studies on propagation of the nuggets in the solar atmosphere have produced very encouraging results [28]. In that work, the detail numerical simulations have been carried out. It has been confirmed that the total energy injected into the corona as a result of the annihilation events of the AQNs with the solar material is order of $10^{27} \mathrm{erg} / \mathrm{s}$. This is very robust prediction of the model in full agreement with observations (1), see Fig. 10 in [28]. This should be considered as a highly nontrivial consistency check of the AQN framework because the original Monte Carlo sample was around $\sim 10^{10}$ particles distributed up to distances $\sim 10$ AU. Furthermore, the annihilation processes effectively started at the altitude $\sim 2200 \mathrm{~km}$ which precisely corresponds to the transition region where some drastic changes are known to occur. The most of the energy is deposited in the transition region, see Fig. 9 in [28], which implies that the axions will be released at the same time at the altitude around $\sim 2200 \mathrm{~km}$.

As we mentioned at the end of Sec. IV B, the present computations at small velocities $v_{a} \ll c$ (which are required to analyze the aforementioned enhancements related to the gravitationally focused DM stream) are not reliable. Therefore, we leave the corresponding estimates with possible enhancement factors for future studies. The only comment we would like to make to conclude this subsection is as follows. As we argued above the streaming dark matter axions may be the better source for their discovery than the widely assumed isotropic DM. This is because, a large axion flux enhancement can take place, temporally, due to gravitational lensing when the Sun and/or a planet are aligned with the stream or an axion caustic pointing to the Earth.

\section{B. AQNs in the Earth}

In this subsection, we want to make a few comments on differences between the AQNs propagation in the solar atmosphere in comparison with the Earth's atmosphere. The drastic changes between the two systems have been previously discussed in [27]. From the theoretical viewpoint, the solar atmosphere is much simpler system which is easier to study than the Earth's atmosphere. The basic reason for such simplification is that the solar corona is a highly ionized system consisting mostly protons and electrons. It should be contrasted with Earth's atmosphere where some atoms (mostly heavy elements $N$ and $O$ ) are neutral and some are partly ionized. The interaction of these heavy neutral elements with the AQNs is a highly complicated problem as the most likely outcome of the collision is the elastic reflection rather than penetration deep inside the nugget with some probability of partial annihilation processes, which inject the energy and produce the axions. The corresponding enhancement factor in the Sun due to long-range Coulomb forces in highly ionized plasma at temperature $T \simeq 10^{6} \mathrm{~K}$ was parametrized in [28] by effective size $R_{\text {eff }} \gg R$ to be distinguished from its physical size $R$. This implies that the effective cross section for protons with AQN in the Sun is approximated as $\sim \pi R_{\text {eff }}^{2}$ while a similar cross section for neutral atoms is $\sim \pi R^{2}$.

As we mentioned above, similar computations have not been carried out for Earth's atmosphere yet. Nevertheless, we would like to make here a few estimates for the axion flux due to the disintegration of the AQNs in the Earth's atmosphere. The AQN flux on the Earth is estimated as follows:

$$
\begin{aligned}
& \frac{d N}{d A d t}=n_{\mathrm{AQN}} v \approx 0.3 \cdot\left(\frac{10^{25}}{\langle B\rangle}\right) \mathrm{km}^{-2} \mathrm{yr}^{-1}, \\
& n_{\mathrm{AQN}} \simeq \frac{\rho_{\mathrm{DM}}}{m_{p}\langle B\rangle} .
\end{aligned}
$$

This tiny rate represents the main reason why the direct detection of the nuggets requires detectors with large area such as Pierre Auger Observatory or Telescope Array to observe the showers produced by the AQNs entering the atmosphere [54,55].

The annihilation processes are much less efficient in the Earth's atmosphere than in the Sun as mentioned above due to the drastic difference between $R$ and $R_{\text {eff }}$ in the ionized hot plasma. It has been estimated in $[35,54,55]$ that only small portion of the AQN's mass $\Delta M \simeq 10^{-10} \mathrm{~kg}$ will get annihilated in the Earth's atmosphere. This represents only tiny portion $\sim \Delta B / B \sim 10^{-8}$ of a typical nugget which can get annihilated in the atmosphere. On entering the earth's crust the nugget will continue to deposit energy along its path, however this energy is dissipated in the surrounding rock and is unlikely to be directly observable. Generally, the nuggets carry sufficient momentum to travel directly through the earth and emerge from the opposite side. However, a finite fraction of the AQNs $(\Delta B / B$ could be around $10 \%$ ) may be captured by the Earth and deposit all their energy in dense regions. ${ }^{3}$

While the observation of the $E \& M$ showers due to the nuggets entering the Earth's atmosphere indeed requires very large area detectors, the observation of the axions (which have been produced as a result of the annihilation events in the very deep underground) is possible, and in fact

\footnotetext{
${ }^{3} \mathrm{~A}$ better estimate requires a precise numerical simulations as different nuggets have different baryon charges $B$, different sizes, different impact velocities. Furthermore, some of the nuggets will have very short path, much shorter than the Earth's radius $R_{\oplus}$, while other nuggets will have long paths of order $\sim 2 R_{\oplus}$, depending on the angle of the impact.
} 
very promising. Indeed, the corresponding axion flux can be estimated from (16) as follows,

$$
\begin{aligned}
m_{a} \Phi(\text { Earth axions }) & \sim \frac{(2 \Delta B) \mathrm{GeV}}{3} \cdot \frac{d N}{d A d t} \\
& \sim 10^{16} \cdot\left(\frac{\Delta B}{B}\right) \frac{\mathrm{eV}}{\mathrm{cm}^{2} \mathrm{~s}},
\end{aligned}
$$

where we assume that $\Delta B \sim B$ charges of the AQNs get annihilated in the earth's core, and each event of annihilation produces $2 \mathrm{GeV}$ energy deep underground. The key point here is that $\sim 1 / 3$ of this energy is radiated in the form of axions similar to our arguments leading to (9). Furthermore, these axions will have the typical velocities $v \sim 0.5 c$ as plotted on Fig. 1. These axions, in principle, can be observed, in contrast with conventional E\&M energy which is dissipated in the mantle or in the Earth's core and completely lost for the direct observations. Notably, for the calculation of (17), we have not taken into account the aforementioned temporally flux enhancement due to planetary (up to $\sim 10^{6}$ ) and in particular solar gravitational focusing (up to $\sim 10^{11}$ ).

Interestingly, the axion flux (17) which is generated due to the AQN annihilation events is much larger than the flux (12) generated due to the AQN annihilation events in the solar corona. At the same time, the axion flux (17) is the same order of magnitude as the conventional cold dark matter galactic axion contribution (13). This is because the parameter $\Delta B / B \sim 1$ is expected to be order of one, as a finite portion of the AQNs will get annihilated in the Earth's core. However, the wave lengths of the axions produced due to AQN annihilations, are much shorter due to their relativistic velocities $v \sim 0.5 c$, in contrast with conventional galactic isotropic axions with $v \sim 10^{-3} c$. Therefore, these two distinct contributions can be easily discriminated.

\section{CONCLUSION AND FUTURE DIRECTIONS}

The main claim of this work is that there is a new mechanism of the axion production (Fig. 1) with drastically different spectral features (in comparison with conventional galactic axions characterized by $v \sim 10^{-3} c$ and the solar axions characterized by typical energies $\langle E\rangle \simeq 4 \mathrm{keV}$ ). The corresponding fluxes are given by Eqs. (17) for the Earth's core and (12) for the solar corona correspondingly. The new mechanism is entirely based on the nonorthodox AQN dark matter model.

Why this new AQN framework (and accompanying the axion emission) should be taken seriously? We refer to Sec. II for overview of this DM model. The only comment we would like to make here is that this model was invented long ago as a natural explanation of the observed ratio (3) between visible and dark matter densities. This model passed all the tests to be qualified as a valid DM candidate. In the context of the present work, the most important feature of this model is that it may potentially resolve the old renowned puzzle (since 1939) known in the community under the name "the solar corona mystery." In particular, this model, without adjusting any parameters, generates the observed EUV luminosity (1) as recent numerical Monte Carlo simulations show [28]. The corresponding intensity $\sim 10^{27} \mathrm{erg} / \mathrm{s}$ is entirely determined by the dark matter density in the solar system. The mysterious temperature inversion around $2000 \mathrm{~km}$ also finds its natural explanation as the most of AQNs inject their energy in this transition region, see Fig. 9 in [28]. Surprisingly, the new axion production leading to the estimate (12) occurs precisely at this transition region.

Following the numerical estimates given above, most DM axion searches (which are presently running, or planning to start taking the data in near future) have the potential to discover DM axions from AQNs which are produced in the solar corona (12) or in the Earth's core (17). The key element which discriminates these axions from conventional DM galactic axions is their wide relativistic velocities at $v \sim 0.5 c$. Therefore, in the present work, we choose not to specify the instruments which are most suitable and capable for such an analysis. Generically speaking, the proper instruments must be either wide-band by default, or, they must implement the fast scanning mode [49,50,52].

In addition, following the planetary dependence of the atmospheric electron density [29], a similar dependence also for a putative DM axion signature from defragmented AQNs seems possible. Such a dependence can be used for signal identification as well as for improving the signal-tonoise ratio resulting to a better detection sensitivity.

\section{ACKNOWLEDGMENTS}

The work of A. Z. and X. L. was supported in part by the Natural Sciences and Engineering Research Council of Canada. H.F., Y.S. and K.Z. would like to thank the CAST Collaboration for inspiring this work. K.Z. thanks Slava Turyshev for encouraging exchanges. K.Z. is partly funded by GSRT Athens/Greece \&ELKE/University of Patras/Greece. The work of Y. S. was supported by IBSR017-D1 of the Republic of Korea.

\section{APPENDIX: TECHNICAL DETAILS. AXION EMISSION FROM THE DOMAIN WALL}

In this Appendix, we want to study the spectral properties of the axion's emission as a result of timedependent perturbations of the axion domain wall. We want to focus on the axion portion of the axion DW, which also includes other fields such as $\pi, \eta^{\prime}$, see [56]. It also contains a phase describing the baryon charge distribution on the surface of the nugget as discussed in [22]. Exact features of the profile functions for all these fields are not important for our purposes. Therefore, one can simplify our computations by considering the 
following effective Lagrangian with two degenerate vacuum states. ${ }^{4}$

$$
\mathcal{S}[\phi]=\int d^{4} x\left[\frac{1}{2}\left(\partial_{\mu} \phi\right)^{2}-\frac{g^{2}}{4}\left(\phi^{2}-\frac{\pi^{2}}{4} f_{a}^{2}\right)^{2}\right],
$$

where $g=\frac{\sqrt{2}}{\pi} \frac{m_{a}}{f_{a}}$, and we set the effective axion angle as $\phi / f_{a} \equiv \theta+\arg \operatorname{det} M+\pi / 2$ (note that we shift the angle by $\pi / 2$ for convenience of calculation). In this toy model, the domain wall solution has an exact form

$$
\phi_{w}=\frac{\pi}{2} f_{a} \tanh \left[\frac{1}{2} m_{a}\left(z-z_{0}\right)\right]
$$

for arbitrary $z_{0}$. Despite the simplicity, this model contains all the essential feature of sine-Gordon Lagrangian we need in this computation. For example, the surface tension of the domain wall in this model is

$$
\sigma \equiv \int_{-\infty}^{\infty}\left(\phi_{w}^{\prime}(z)\right)^{2}=\frac{\pi^{2}}{6} f_{a}^{2} m_{a}
$$

which is very similar to the classic value $\sigma \simeq 8 f_{a}^{2} m_{a}$ computed for the sine-Gordon potential.

Our goal now is to compute the excitations $\chi(t, z)$ in the time-dependent background. These excitations will be eventually identified with the axions emitted by the axion DW. To achieve this task, we expand $\phi(t, z)=$ $\phi_{w}\left(z-R_{0}\right)+\chi(t, z)$, which gives

$$
\begin{aligned}
\mathcal{S}[\phi]= & \mathcal{S}\left[\phi_{w}\right]+\int d t \int d^{2} x_{\perp} \int d z\left[\frac{1}{2} \dot{\chi}^{2}-\frac{1}{2} \chi L_{2} \chi\right] \\
& +\mathcal{O}\left(\chi^{3}\right)
\end{aligned}
$$

where $L_{2}$ is a linear differential operator of the second order,

$$
\begin{aligned}
L_{2} & =\left.\left[-\frac{\partial^{2}}{\partial z^{2}}+2 g^{2} \phi^{2}+g^{2}\left(\phi^{2}-v^{2}\right)\right]\right|_{\phi=\phi_{w}\left(z-R_{0}\right)} \\
& =-\frac{\partial^{2}}{\partial z^{2}}+\frac{m^{2}}{2}\left[3 \tanh ^{2}\left(\frac{1}{2} m_{a}\left(z-R_{0}\right)\right)-1\right] .
\end{aligned}
$$

\footnotetext{
${ }^{4}$ In our previous studies [22-24], we always discussed the socalled $N=1$ domain walls. It implies that the vacuum is unique and the DW solution interpolates between one and the same physical vacuum. This interpolation always occurs as a result of variation of the axion field together with another fields, such as $\pi$ or $\eta^{\prime}$ as discussed in [56]. These additional fields do not generate much changes in the domain wall tension, nor they affect our analysis of the axion production, which is the subject of the present work. Therefore, we ignore these fields to simplify notations and qualitative analysis in this work.
}

The corresponding equation of motion is, therefore,

$$
\frac{\partial^{2}}{\partial t^{2}} \chi=-L_{2} \chi
$$

To look for the initial conditions, we now want to describe the emission of axions in one cycle of oscillation. As mentioned in Sec. IV B, annihilation of baryon charge results in oscillations of domain wall. Assuming the oscillation is approximately adiabatic, it is sufficient to only analyze the first half of an oscillation-say, the "contraction period"-where the domain wall shrinks from $R_{0}$ to a slightly smaller size $R_{0}-\Delta R$. We assumed the rest half of the cycle, the "expansion period," is just the timereversed and produces an equivalent contribution. We may write down such initial conditions as

$$
\phi(0, z)=\phi_{w}\left(z-R_{0}\right)
$$

$\phi\left(\frac{1}{2} t_{\mathrm{osc}}, z\right)=\phi_{w}\left(z-R_{0}+\Delta R\right)+($ excitations $)$,

where $t_{\mathrm{osc}}$ denotes the period of one full oscillation. The excitation modes in condition (A7b) is unknown and depends on the conversion rate from excitation modes to freely propagating axions. In terms of $\chi$, the initial conditions (A7) imply

$$
\begin{aligned}
\chi(0, z) & =0, \\
\chi\left(\frac{1}{2} t_{\mathrm{osc}}, z\right) & \simeq \sqrt{\eta} \phi_{w}^{\prime}\left(z-R_{0}\right) \Delta R+\mathcal{O}\left(\Delta R^{2}\right),
\end{aligned}
$$

where we introduce a free parameter $\eta$ to account for the conversion rate to axion radiation, so $\eta$ varies from 0 to 1 . An efficient conversion corresponds to $\eta \sim 1$, and a poor rate of conversion corresponds to $\eta \ll 1$. In general, we should expect $\eta \sim 1$. This numerical factor does not modify our conclusion about the spectrum. It may only affect the intensity which is fixed by the observed EUV emission, and it is given by Eq. (10).

We now express $\chi$ in term of normalized basis

$$
\begin{aligned}
& \chi(t, z)=\int_{-\infty}^{\infty} d p a_{p}(t) \chi_{p}(z), \\
& \chi_{p}(z) \equiv \frac{1}{\sqrt{4 \pi E_{a} S}} e^{i p z} .
\end{aligned}
$$

Note that $L_{2}$ is diagonal in the basis $\chi_{p}$ 


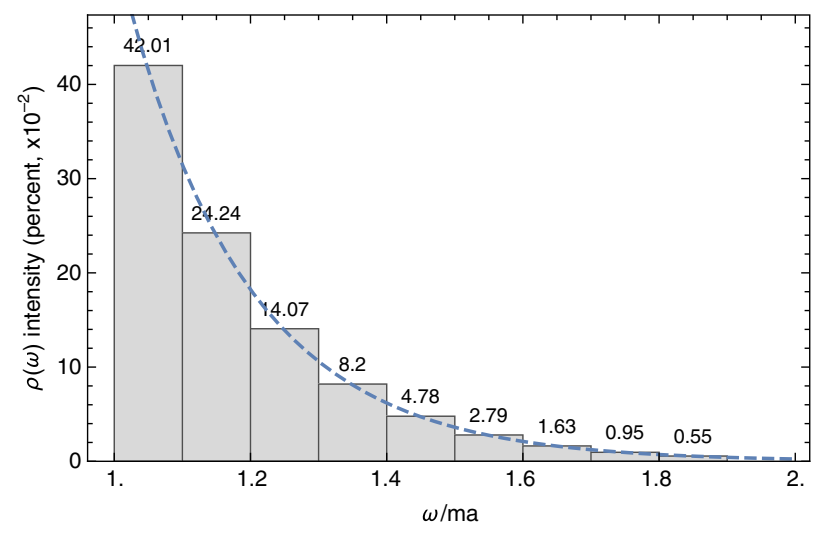

(a)

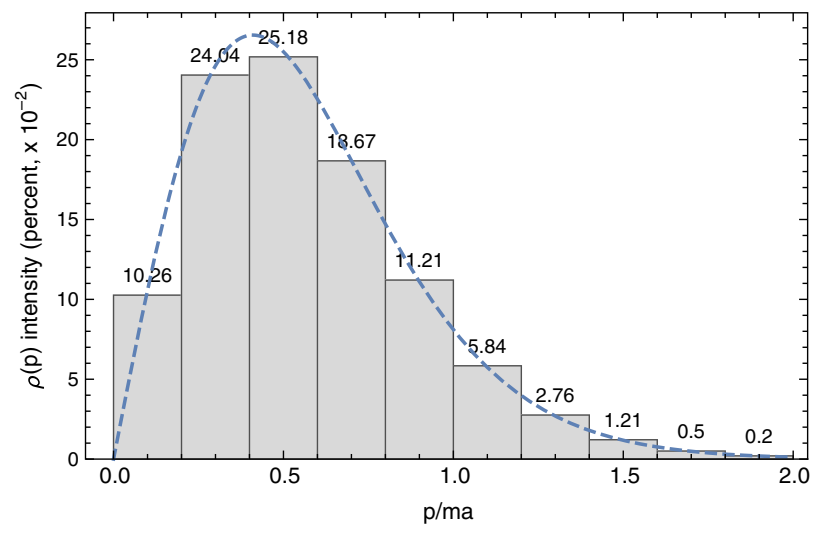

(b)

FIG. 2. (Normalized) flux spectrum for energy and momentum. (a) The normalized spectrum $\rho\left(E_{a}\right)$ vs $\omega$. (b) The normalized spectrum $\rho(p)$ vs $p$.

$$
\begin{aligned}
\int d^{3} x \chi_{p}^{*}(z) L_{2} \chi_{q}(z) & =\frac{1}{4 \pi E_{a}} \int_{-\infty}^{\infty} d z e^{-i(p-q) z}\left\{q^{2}+\frac{m_{a}^{2}}{2}\left[3 \tanh ^{2}\left(\frac{1}{2} m_{a}\left(z-R_{0}\right)-1\right)\right]\right\} \\
& =\frac{p^{2}}{2 E_{a}} \delta(p-q)+K_{p, q} \frac{m_{a}^{2}}{4 \pi E_{a}} e^{-i(p-q) R_{0}} \int_{-\infty}^{\infty} d z e^{-i(p-q) z} \\
& =\frac{\delta(p-q)}{2 E_{a}}\left(p^{2}+K_{p, q} m_{a}^{2}\right)
\end{aligned}
$$

where in the intermediate step, we have defined the ratio

$$
K_{p, q} \equiv \frac{\int_{-\infty}^{\infty} d z e^{-i[p-q-\operatorname{sign}(z) \cdot i \varepsilon] z} \frac{1}{2}\left[3 \tanh ^{2}\left(\frac{1}{2} m_{a} z\right)-1\right]}{\int_{-\infty}^{\infty} d z e^{-i[p-q-\operatorname{sign}(z) \cdot i \varepsilon] z}}
$$

for simplicity of calculation. Note that $K_{p, q}$ is finite and well defined in the entire range of $p, q$. For our computations when $p=q$ the parameter $K_{p, q}=1$ as $K_{p, q} \delta(p-q)=\delta(p-q)$. Then Eq. (A10) is simplified to

$$
\int d^{3} x \chi_{p}^{*}(z) L_{2} \chi_{q}(z)=\frac{\delta(p-q)}{2 E_{a}}\left(p^{2}+m_{a}^{2}\right) .
$$

Our original Eq. (A6) now can be simplified into

$$
\frac{d^{2}}{d t^{2}} a_{p}=-E_{a}^{2}(p) a_{p}, \quad E_{a}(p) \equiv \sqrt{p^{2}+m_{a}^{2}}
$$

Solving Eq. (A13) with initial conditions (A8) gives

$$
\begin{aligned}
a_{p}(t)= & e^{-i p R_{0}} \Delta R \frac{\pi}{2} \frac{f_{a}}{m_{a}} \sqrt{4 \pi \eta S E_{a}} \frac{\sin E_{a} t}{\sin \left(\frac{1}{2} E_{a} t_{\mathrm{osc}}\right)} \\
& \times p \operatorname{csch}\left(\frac{\pi p}{m_{a}}\right) .
\end{aligned}
$$

Then, the total radiation energy of the domain wall is obviously

$$
\begin{aligned}
E_{\mathrm{rad}}= & \int d^{3} x \frac{1}{2} \chi^{*}\left[-\frac{\partial^{2}}{\partial t^{2}}+L_{2}\right] \chi \\
= & \int_{-\infty}^{\infty} d p \frac{1}{2} E_{a}(p)\left|a_{p}\right|^{2} \\
= & \int_{m_{a}}^{\infty} d E_{a} \pi^{3} \eta S \Delta R^{2}\left(\frac{f_{a}}{m_{a}}\right)^{2}\left[\frac{\sin E_{a} t}{\sin \left(\frac{1}{2} E_{a} t_{\mathrm{osc}}\right)}\right]^{2} \\
& \times E_{a}^{3} p \operatorname{csch}^{2}\left(\frac{\pi p}{m_{a}}\right) .
\end{aligned}
$$

More generally, the domain wall is oscillating in a shallow cavity $S \Delta R$, so the excitation energy density (in volume) is $E_{\text {rad }} / S \Delta R$. Then the axion flux spectrum $\Phi_{\text {rad }}$ emitted from a single $A Q N$ is clearly

$$
\begin{aligned}
\frac{1}{S} \frac{d}{d E_{a}} \Phi_{\mathrm{rad}}= & \frac{p}{E_{a}^{2}} \frac{d}{d E_{a}}\left(\frac{E_{\mathrm{rad}}}{S \Delta R}\right) \\
= & \pi^{3} \eta \Delta R\left(\frac{f_{a}}{m_{a}}\right)^{2}\left[\frac{\sin E_{a} t}{\sin \left(\frac{1}{2} E_{a} t_{\mathrm{osc}}\right)}\right]^{2} \\
& \times E_{a} p^{2} \operatorname{csch}^{2}\left(\frac{\pi p}{m_{a}}\right) .
\end{aligned}
$$


Such spectrum indicates an average energy $\left\langle E_{a}\right\rangle=1.18 m_{a}$. One may also see Fig. 2, where the normalized flux spectra as a function of $E_{a}$ and $p$ are plotted in Fig. 2. It is also useful to obtain the spectrum as a function of axion velocity $v_{a}$,

$$
\begin{aligned}
\frac{1}{S} \frac{d}{d v_{a}} \Phi_{\mathrm{rad}}= & \pi^{3} \eta \Delta R\left(\frac{f_{a}}{m_{a}^{2}}\right)^{2}\left[\frac{\sin E_{a} t}{\sin \left(\frac{1}{2} E_{a} t_{\mathrm{osc}}\right)}\right]^{2} \\
& \times E_{a}^{3} p^{3} \operatorname{csch}^{2}\left(\frac{\pi p}{m_{a}}\right),
\end{aligned}
$$

which gives Fig. 1 in Sec. IV B.

We conclude this Appendix with the following comments. The main goal of this analysis is the computation of the spectrum which is plotted above. The intensity of the radiation is determined by our expressions (9) and (10) which are based on assumption that the travel time of the AQN in the solar corona is sufficiently long. Therefore, the total charge of the antinuggets will be completely annihilated, and the total intensity of the axion emission is fixed and given by (9) and (10). The numerical analysis carried out in [28] supports this assumption as most of the nuggets indeed get annihilated at the altitude around $2000 \mathrm{~km}$.

The analysis presented above suggests that the typical velocities of the emitted axions $v_{a} \simeq 0.5 c$. This is an expected result because the energies of the emitted axions are determined by the moving domain wall which normally have velocities close to the speed of light, i.e., $\Delta R \sim t_{\mathrm{osc}}$. Precisely this condition eventually determines the spectral density of the emitted axions.

The basic picture of the emission developed in this Appendix is based on the thin wall approximation when infinitely large (along $x, y$ directions) DW moves with acceleration and emits axion waves moving along $z$ direction. It is quite obvious that this approach is not justified when de Broglie wavelength $\lambda_{a}$ is comparable with the thickness of the domain wall as explained in Sec. IV B. It implies that the small velocity portion of the spectrum may receive large corrections as a result of break down of the thin wall approximation. Linear dependence on the velocity at small $v_{a} \ll c$ is manifestation of this approximation when the system can be shifted along $x, y$ directions without changing the system.
[1] R. D. Peccei and H. R. Quinn, Phys. Rev. D 16, 1791 (1977); S. Weinberg, Phys. Rev. Lett. 40, 223 (1978); F. Wilczek, Phys. Rev. Lett. 40, 279 (1978).

[2] J. E. Kim, Phys. Rev. Lett. 43 (1979) 103; M. A. Shifman, A. I. Vainshtein, and V. I. Zakharov, Nucl. Phys. B166, 493 (1980).

[3] M. Dine, W. Fischler, and M. Srednicki, Phys. Lett. B 104, 199 (1981); A. R. Zhitnitsky, Yad. Fiz. 31, 497 (1980); [Sov. J. Nucl. Phys. 31, 260 (1980)].

[4] K. van Bibber and L. J. Rosenberg, Phys. Today 59, No. 8, 30 (2006).

[5] S. J. Asztalos, L. J. Rosenberg, K. van Bibber, P. Sikivie, and K. Zioutas, Annu. Rev. Nucl. Part. Sci. 56, 293 (2006).

[6] P. Sikivie, Lect. Notes Phys. 741, 19 (2008).

[7] G. G. Raffelt, Lect. Notes Phys. 741, 51 (2008).

[8] P. Sikivie, Int. J. Mod. Phys. A 25, 554 (2010).

[9] L. J. Rosenberg, Proceedings of the National Academy of Sciences of India, Section A (Physical Sciences) 112, 12278 (2015).

[10] P. W. Graham, I. G. Irastorza, S. K. Lamoreaux, A. Lindner, and K. A. van Bibber, Annu. Rev. Nucl. Part. Sci. 65, 485 (2015).

[11] D. J. E. Marsh, Phys. Rep. 643, 1 (2016).

[12] A. Ringwald, Proc. Sci. NOW2016 (2016) 081 [arXiv: 1612.08933].

[13] J. Preskill, M. B. Wise, and F. Wilczek, Phys. Lett. B 120, 127 (1983); L. Abbott and P. Sikivie, Phys. Lett. B 120, 133 (1983); M. Dine and W. Fischler, Phys. Lett. B 120, 137 (1983).
[14] S. Chang, C. Hagmann, and P. Sikivie, Phys. Rev. D 59, 023505 (1998).

[15] T. Hiramatsu, M. Kawasaki, K. Saikawa, and T. Sekiguchi, Phys. Rev. D 85, 105020 (2012); 86, 089902(E) (2012).

[16] M. Kawasaki, K. Saikawa, and T. Sekiguchi, Phys. Rev. D 91, 065014 (2015).

[17] L. Fleury and G. D. Moore, J. Cosmol. Astropart. Phys. 01 (2016) 004.

[18] V. B. Klaer and G. D. Moore, J. Cosmol. Astropart. Phys. 11 (2017) 049.

[19] P. Sikivie, Phys. Rev. Lett. 51, 1415 (1983); 52, 695(E) (1984).

[20] V. Anastassopoulos et al. (CAST Collaboration), Nat. Phys. 13, 584 (2017).

[21] A. R. Zhitnitsky, J. Cosmol. Astropart. Phys. 10 (2003) 010.

[22] X. Liang and A. Zhitnitsky, Phys. Rev. D 94, 083502 (2016).

[23] S. Ge, X. Liang, and A. Zhitnitsky, Phys. Rev. D 96, 063514 (2017).

[24] S. Ge, X. Liang, and A. Zhitnitsky, Phys. Rev. D 97, 043008 (2018).

[25] J. A. Klimchuk, Sol. Phys. 234, 41 (2006).

[26] L. DiLella and K. Zioutas, Astropart. Phys. 19, 145 (2003).

[27] A. Zhitnitsky, J. Cosmol. Astropart. Phys. 10 (2017) 050.

[28] N. Raza, L. van Waerbeke, and A. Zhitnitsky, Sol. Phys. 234, 41 (2006).

[29] S. Bertolucci, K.Zioutas, S. Hofmann, and M. Maroudas, Phys. Dark Universe 17, 13 (2017).

[30] A. Zhitnitsky, J. Cosmol. Astropart. Phys. 10 (2017) 050. 
[31] E. Witten, Phys. Rev. D 30, 272 (1984).

[32] E. Farhi and R. L. Jaffe, Phys. Rev. D 30, 2379 (1984).

[33] A. De Rujula and S. L. Glashow, Nature (London) 312, 734 (1984).

[34] J. Madsen, Lect. Notes Phys. 516, 162 (1999).

[35] K. Lawson and A. R. Zhitnitsky, in Proceedings of Snowmass 2013 [arXiv:1305.6318].

[36] D. M. Jacobs, G. D. Starkman, and B. W. Lynn, Mon. Not. R. Astron. Soc. 450, 3418 (2015).

[37] A. D. Sakharov, JETP Lett. 5, 24 (1967).

[38] A. Zhitnitsky, Phys. Rev. D 74, 043515 (2006).

[39] D. H. Oaknin and A. R. Zhitnitsky, Phys. Rev. Lett. 94, 101301 (2005).

[40] A. Zhitnitsky, Phys. Rev. D 76, 103518 (2007).

[41] J. D. Bowman, A. E. E. Rogers, R. A. Monsalve, T. J. Mozdzen, and N. Mahesh, Nature (London) 555, 67 (2018).

[42] K. Lawson and A. R. Zhitnitsky, arXiv:1804.07340.

[43] E. N. Parker, Astrophys J. 264, 642 (1983); 330, 474 (1988).

[44] J. A. Klimchuk (Hinode Review Team), arXiv:1709.07320.

[45] E. A. Bruevich and G. V. Yakunina, arXiv:1711.06262.

[46] K. Shibata and S. Takasao, arXiv:1606.09401.

[47] K. Shibata et al., Science 318, 1591 (2007).
[48] Y. K. Semertzidis, http://capp.ibs.re.kr/html/capp_en/CAPP_ research_plan_20180306.pdf.

[49] K. Zioutas, V. Anastassopoulos, S. Bertolucci, G. Cantatore, S. A. Cetin, H. Fischer, W. Funk, A. Gardikiotis, D. H. H. Hoffmann, S. Hofmann, M. Karuza, M. Maroudas, Y. K. Semertzidis, and I. Tkatchev, arXiv:1703.01436.

[50] H. Fischer, Y. K. Semertzidis, and K. Zioutas, https:// ep-news.web.cern.ch/content/search-axions-streaming-darkmatter.

[51] B. R. Patla, R. J. Nemiroff, D. H. H. Hoffmann, and K. Zioutas, Astrophys. J. 780, 158 (2014).

[52] K. Zioutas, in Proceedings of Axion Dark Matter Conference, NORDITA, Stockholm 2016, edited by F. Wilczek, S. Baum, A. Goobar, L. Bergstrom, K. Freese, and Y. Semertzidis (AlbaNova, Stockholm), https://www .nordita.org/docs/agenda/slides-axions2016-zioutas.pdf.

[53] S. G. Turyshev, Phys. Rev. D 95, 084041 (2017); S. G. Turyshev and V. T. Toth, Phys. Rev. D 96, 024008 (2017).

[54] K. Lawson, Phys. Rev. D 83, 103520 (2011).

[55] K. Lawson, Phys. Rev. D 88, 043519 (2013).

[56] M. M. Forbes and A. R. Zhitnitsky, J. High Energy Phys. 10 (2001) 013. 